\title{
An innovative approach for HLA typing, molecular tumor testing and the validation of tumor exclusive antigens
}

Michael Ghosh, Leon Bichmann, Jonas Scheid, Gizem Güler, Heiko Schuster, Moreno Di Marco, Ana Marcu, Meret Beyer, Annika Nelde, Lena K. Freudenmann, Lena Mühlenbruch, Markus W. Löffler, Oliver Kohlbacher, Hans-Georg Rammensee, Stefan Stevanović

Correspondence to: michaelghosh@uni-tuebingen.de

\section{Disclaimer}

This manuscript has been withdrawn by the corresponding author, as it was submitted and made public in bioRxiv without knowledge and the full consent of all the authors listed. The co-authors are therefore not responsible for the contents of this manuscript. For this reason, the submitting author has chosen to withdraw this preprint as a precautionary measure and would like to state that this work should not be cited as a reference. For any related questions that may arise, please contact the corresponding author. 